\title{
Editorial
}

\section{Patellofemoral Pain: Symptom or Disease?}

\author{
Giuseppe Milano ${ }^{1}$ \\ ${ }^{1}$ Department of Orthopaedics, Catholic University, Rome, Italy
}

Joints 2018;6:73-74.

Two clinical studies published in this issue of Joints deal with the complex issue of patellofemoral pain treatment. Enea et al ${ }^{1}$ report the results obtained using distalization and medialization of the tibial tuberosity, a surgical treatment for potential patellar instability (PPI) associated with a particularly "challenging" predisposing factor, namely, the patella alta. The index group (PPI group), consisting of patients with pain but no episodes of frank patellar instability, was compared with a group of patients reporting pain and objective patellar instability. The authors found that the treatment significantly reduced pain and improved knee function in both groups. However, the improvement in subjective instability was significantly more marked in the patients with PPI. In short, the proposed treatment was found to be particularly effective in the treatment of patients with PPI and patellofemoral pain.

The article by Uboldi et $\mathrm{al}^{2}{ }^{2}$ concerns a prospective randomized trial on the efficacy of an elastomeric knee brace used in the treatment of patellofemoral pain syndrome (PFPS) in adjunct to a specifically developed rehabilitation program. Function was found to be significantly improved both in the study group and in the controls (who only received rehabilitation); however, use of the knee brace was associated with significantly reduced pain.

Patellofemoral pain is, undoubtedly, a clinical problem with a high social and economic impact, given that it affects a young and active section of the population. Indeed, in addition to rehabilitation treatment costs, it is also necessary to consider the costs related to reduced productivity, due to days off work, and reduced work efficiency.

Undoubtedly, surgery, when indicated, is effective in resolving symptoms. However, in most cases, the pain in PFPS is disabling, and surgical treatment, however adequately it corrects the factors underlying the clinical presentation, is rather invasive and not always predictable in terms of late sequelae. There have been few rigorous analyses of exercise therapy for the treatment of PFPS. A Cochrane review $^{3}$ has highlighted the effectiveness of rehabilitation treatment in helping to reduce knee pain and function in patients with PFPS. Tan et $\mathrm{al}^{4}$ showed that annual direct medical costs are higher in patients receiving rehabilitation treatment compared with those not receiving treatment, although the latter generate higher social costs, in particular, deriving from loss of productivity and work efficiency. Overall, therefore, rehabilitation treatment of PFPS has a favorable cost-effectiveness ratio.

But what are we treating when we treat PFPS? A symptom or a disease? I certainly have no intention of wading into the complex debate regarding the nomenclature and classification of all those clinical situations in which patellofemoral pain, or anterior knee pain as it may more generally be defined, can be recognized as a component. However, it is certainly true that patellofemoral pain belongs to a category of chronic osteoarticular pain that, over the years, has been the focus of a proliferation of therapeutic approaches aimed primarily at treating the symptom rather than its cause, thereby interpreting pain as a disease per se, rather than a sign of a problem. I am thinking, in particular, of lower back pain, a clinical condition that nowadays often seems to be dealt independently of its underlying organic cause, and that has attracted considerable attention from pain therapists aiming to address chronic pain in a multidisciplinary framework in which certain neuropathological mechanisms underlying pain, such as central sensitization, cortical reorganization, neuroimmune dysregulation, and sympathetic nervous system upregulation, are seen as possible treatment targets. Even the biopsychosocial approach has been introduced into the multimodal management of chronic low back pain. A Medline search using the terms "biopsychosocial \& low back pain" will identify as many as 250 articles that approach the problem of low back pain from a sociocultural perspective. Today, however, we can only wonder what became of the "bio" part of chronic pain treatment, ${ }^{5}$ while fortunately, or unfortunately, use of the biopsychosocial approach in the field of patellofemoral pain is limited to a single study. ${ }^{6}$

We must certainly avoid accepting, uncritically, the idea that "pain equals damage"; after all, were this the case, it would be impossible to understand why chronic pain is not constantly

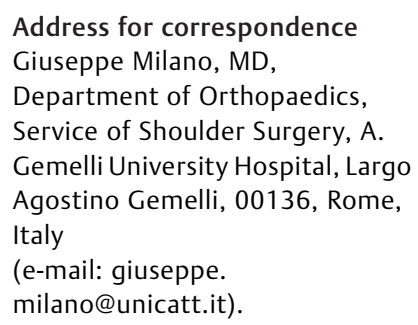

DOI https://doi.org/ 10.1055/s-0038-1667195. ISSN 2282-4324.
Copyright (c) 2018 Georg Thieme Verlag License terms KG Stuttgart - New York 
and consistently unbearable! As Patrick Wall pointed out 30 years ago, "the labeling of nociceptors as pain fibers was not an admirable simplification, but an unfortunate trivialization under the guise of simplification." 7 However, if we allow pain to become a disease and no longer a symptom, we risk neglecting the primary source of the alarm signal that our body is sending out, with the consequent risk of allowing the development of a chronic disease with a subclinical course or, even worse, a silent but devastating worsening of anatomical damage at the site of origin of the pain.

\section{References}

1 Enea D, Canè PP, Fravisini M, Gigante A, Dei Giudici L. Distalization and medialization of tibial tuberosity for the treatment of potential patellar instability with patella alta. Joints 2018;6(02):80-84
2 Uboldi FM, Ferrua P, Tradati D, et al. Use of an elastomeric knee brace in patellofemoral pain syndrome: short-term results. Joints 2018;6(02):85-89

3 Heintjes E, Berger MY, Bierma-Zeinstra SM, Bernsen RM, Verhaar JA, Koes BW. Exercise therapy for patellofemoral pain syndrome. Cochrane Database Syst Rev 2003;(04):CD003472

4 Tan SS, van Linschoten RL, van Middelkoop M, Koes BW, BiermaZeinstra SM, Koopmanschap MA. Cost-utility of exercise therapy in adolescents and young adults suffering from the patellofemoral pain syndrome. Scand J Med Sci Sports 2010;20(04): 568-579

5 Hancock MJ, Maher CG, Laslett M, Hay E, Koes B. Discussion paper: what happened to the 'bio' in the bio-psycho-social model of low back pain? Eur Spine J 2011;20(12):2105-2110

6 Smith BE, Moffatt F, Hendrick P, et al. The experience of living with patellofemoral pain-loss, confusion and fear-avoidance: a UK qualitative study. BMJ Open 2018;8(01):e018624

7 Wall P, McMahon S. The relationship of perceived pain to afferent nerve impulses. Trends Neurosci 1986;9(06):254-255 